\title{
PREVENÇÃO E CONTROLE DE INFECÇÕES POR CORONAVÍRUS EM INSTITUIÇÕES DE LONGA PERMANÊNCIA PARA IDOSOS
}

\author{
Tânia Maria de Oliva Menezes' \\ ORCID: 0000-0001-5819-0570 \\ Romulo Delvalle" \\ ORCID: 0000-0002-8782-6184
}

Ana Maria Ribeiro dos Santos"I

ORCID: 0000-0002-5825-5335

Josiane Steil Siewert ${ }^{\text {IV }}$

ORCID: 0000-0002-8952-2360

'Universidade Federal da Bahia. Salvador, Bahia, Brasil.

" Ministério Público do Estado do Rio de Janeiro. Rio de Janeiro, Rio de Janeiro, Brasil.

"'Universidade Federal do Piauí. Teresina, Piauí, Brasil.

IVInstituto Federal de Educação, Ciência e Tecnologia de Santa Catarina. Joinville, Santa Catarina, Brasil.

Autor Correspondente: Tânia Maria de Oliva Menezes E-mail: tomenezes50@gmail.com

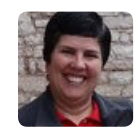

Como citar

Menezes TMO, Delvalle R, Santos AMR, Siewert JS. Prevenção e controle de infecções por coronavírus em instituições de longa permanência para idosos.

In: Santana RF. Enfermagem gerontologica no cuidado do idoso em tempos da COVID 19.

2.ed.rev. Brasilia, DF: Editora ABEn; 2020. p.17-22.

(Serie Enfermagem e Pandemias, 1). https://doi.org/10.51234/aben.20.e01.c03

\section{INTRODUÇÃO}

A Organização Mundial de Saúde (OMS), em reconhecimento à ampla transmissão global da COVID-19, declarou pandemia em 11 de março de 2020(1). As Instituições de Longa Permanência para Idosos (ILPIs) são ambientes de alto risco para resultados graves de surtos de COVID-19, devido à idade avançada e às condições de saúde crônicas frequentes dos residentes, bem como à movimentação de profissionais de saúde entre as instalações de uma região(2).

Impedir a disseminação do COVID-19 nas ILPIs é uma prioridade e devem ser tomadas medidas rigorosas para garantir isso ${ }^{(3)}$. Prevenção e controle de infecção devem ser implementadas pelos profissionais que atuam nos serviços de saúde, para evitar ou reduzir ao máximo a transmissão de microrganismos durante qualquer assistência à saúde realizada. A melhor maneira de prevenir a COVID-19 é adotar ações para impedir a propagação do vírus, e o serviço de saúde deve garantir que as políticas e as boas práticas internas minimizem a exposição a patógenos respiratórios, incluindo o novo coronavírus (SARS-CoV-2) ${ }^{(4)}$.

As ILPI devem implementar medidas de prevenção e controle de infecção para evitar ou reduzir ao máximo que os residentes, seus cuidadores e profissionais que atuem nesses estabelecimentos sejam infectados pelo vírus e, mais significativamente, reduzir a morbi-mortalidade entre os idosos nessas instituições ${ }^{(5)}$.

Alguns fatores contribuem para a vulnerabilidade nestas instituições como: funcionários que trabalham quando sintomáticos; funcionários que trabalham em mais de uma ILPI; familiaridade e aderência inadequadas às recomendações de Equipamentos de Proteção Individual (EPI); desafios para a implementação de práticas adequadas de controle de infecção, incluindo suprimentos inadequados de EPI e outros itens, a exemplo, desinfetante para as mãos à base de álcool; atraso no reconhecimento dos casos devido a um baixo índice de 
suspeita; disponibilidade limitada de testes; e dificuldade em identificar pessoas com Covid-19 com base apenas em sinais e sintomas ${ }^{(2)}$.

Diante deste cenário, o Departamento Científico de Enfermagem Gerontológica, da Associação Brasileira de Enfermagem, ao reconhecer a grave situação que o Brasil enfrenta e a intensidade com que a Enfermagem vem sendo afetada no contexto dessa pandemia, discute medidas que contribuem para a prevenção e controle no cotidiano das ILPIs.

\section{OBJETIVO}

Discutir sobre a prevenção e controle de infecções por coronavírus em instituições de longa permanência para idosos.

\section{MÉTODO}

Ensaio teórico de natureza reflexiva, baseado em artigos científicos nacionais, internacionais e portarias.

\section{RESULTADOS}

A chegada do profissional a ILPI, sua movimentação em todo o ambiente e a saída da instituição deve ser monitorada pelo gestor, de forma a garantir segurança para os residentes e trabalhadores. Portanto, orientar o profissional com regularidade para uso de máscara, cirúrgica ou de tecido, durante todo o percurso até chegar a ILPI, pode ser uma primeira medida à ser adotada.

\section{Profilaxia adotada pelos funcionários ao chegar às instituições}

A prevenção e controle de infecção pelo coronavírus iniciam na porta de entrada da instituição. Impedir a disseminação da COVID-19 para instituições de assistência a longo prazo é uma prioridade e devem ser tomadas medidas rigorosas para garantir isso, começando com a chegada dos profissionais a ILPI ${ }^{(3)}$.

Na entrada da ILPI, fixar cartazes com orientações sobre os cuidados necessários como: higienização das mãos, etiqueta respiratória e importância de não entrar na ILPI se apresentar algum sintoma Na triagem ativa, alguns aspectos devem ser observados:

- Destacar um profissional de saúde, preferencialmente enfermeiro, para realizar a triagem ativa de todos os profissionais que forem entrar na ILPI. A triagem deve ser feita em todas as mudanças de plantão. É obrigatória e deve ser feita diariamente, até que seja declarado fim da pandemia no território nacional.

- Disponibilizar lavatório com água e sabão, ou álcool em gel a 70\% no ambiente da triagem. Dispor de lixeiras acionadas por pedal, para o descarte do lixo comum.

- Paramentar o profissional para a realização da triagem com: máscara cirúrgica, touca, capote, sapato fechado, óculos de proteção, protetor facial e luvas de procedimento.

- Evitar aglomeração de pessoas no local de triagem, respeitando as regras de distanciamento.

- Realizar desinfecção do termômetro a cada utilização, com álcool a 70\%.

- Realizar desinfecção do ambiente utilizado após a realização da triagem.

\section{Roteiro de triagem}

1- Aferir a temperatura do profissional. Considerar febril se temperatura maior que $37,5^{\circ} \mathrm{C}$.

( ) SIM ( ) NÃO 
2- Apresenta algum desses sintomas: febre, tosse, dor de garganta, coriza e dificuldade respiratória?

( ) SIM ( ) NÃO

3- Você teve contato nos últimos dias com pessoas com suspeita ou confirmada de COVID-19?

( ) SIM ( ) NÃO

Caso o funcionário responda SIM para qualquer uma das perguntas, orientá-lo a retornar para a sua casa e realizar quarentena, disponibilizar máscara, para que o funcionário retorne para casa com segurança, e apoiá-lo emocionalmente.

O profissional terá acesso a ILPI caso tenha respondido NÃO para as três perguntas do questionário. Em seguida, encaminhar o profissional para a área de descontaminação, independente do local onde estão permanecem os idosos naquele momento. Nessa área, o funcionário irá realizar:

- Retirada de todos os adornos das mãos

- Preensão dos cabelos

- Higienização das mãos com água e sabão, com técnica correta

- Mudança de roupas e calçados, para iniciar os trabalhos.

- Descontaminação dos óculos de grau com álcool a 70\%, caso o profissional utilize.

- Realizar o mesmo procedimento de descontaminação do aparelho celular desligado.

Após estes cuidados, o profissional está apto a iniciar suas atividades laborais na ILPI. Ao término de utilização da área de descontaminação, é importante à higienização do ambiente pelo serviço de limpeza, com água e solução de hipoclorito de sódio em todas as superfícies (maçanetas, portas, cadeiras, interruptor).

O profissional deve ter cuidado com as vestimentas ao entrar e sair da instituição, evitando adentrar áreas internas com roupas usadas na rua. Se possível, prover mudas diárias de roupas pessoais limpas para uso na instituição(6).

Essas etapas devem ser seguidas obrigatoriamente por todos os funcionários diariamente. Essas medidas irão minimizar a propagação da COVID-19 na ILPI, e não devem ser flexionadas e ou diminuídas em nenhum momento. Os cuidadores e demais profissionais deverão utilizar máscara cirúrgica durante o tempo em que estiver na ILPI, atento a periodicidade da troca, conforme recomendações. Na saída, os funcionários devem ser orientados para que o calçado seja de uso exclusivo na ILPI, devendo ter um local para que seja guardado e utilizado no próximo turno.

\section{O ambiente da Instituição}

O serviço de saúde deve garantir que as políticas e as boas práticas internas minimizem a exposição a patógenos respiratórios, incluindo o novo coronavírus ${ }^{(4)}$. A gestão da ILPI deve garantir a limpeza correta e frequente, diariamente e sempre que necessário, das superfícies das áreas comuns, dos dormitórios (pelo menos duas vezes ao dia) e de outros ambientes utilizados pelos idosos ${ }^{(5)}$.

Além disso, deve prover os equipamentos e insumos necessários para a correta higiene das mãos como ${ }^{(4,6)}$ :

- Lavatório/pia com dispensador de sabonete líquido e/ou álcool em gel 70\%

- Suporte para papel toalha e toalha

- Lixeira com tampa e abertura sem contato manual

- Material de limpeza para higienizar superfícies e objetos

Em locais de maior circulação devem ser fixados cartazes informativos sobre a importância das medidas de higiene e distanciamento. Os ambientes devem ser mantidos ventilados, se possível, com as janelas abertas ${ }^{(4)}$. Orientar para que se evite a concentração de residentes em espaços não arejados ${ }^{(6)}$. É fundamental que as 
instituições estabelecem escalas e horários para a saída dos idosos dos quartos e locomoção em áreas comuns, banhos de sol e refeições, reduzindo o tempo dos residentes nas áreas comuns da instituição, para evitar aglomerações ${ }^{(5)}$. É importante alterar a dinâmica das refeições, definindo grupos menores de idosos para ir ao refeitório em momentos diferentes, de modo a respeitar a distância de 1 a 2 metros entre os residentes.

A rotina de limpeza e higienização de espaços e superfícies deve ser intensificada, devendo os profissionais responsáveis enfatizar a higienização de maçanetas, corrimãos, mesas e cadeiras de uso comum ${ }^{(6)}$. A socialização dos idosos deve ser considerada e estimulada, entretanto, a dinâmica também deve ser modificada, promovendo atividades com grupos menores e respeitando a distância mínima entre eles.

\section{Planejamento dos quartos para isolamento dos casos suspeitos/confirmados}

Com o avanço do COVID-19 no Brasil, proteger os idosos que vivem em Instituições de Longa Permanência para Idosos se tornou uma prioridade às autoridades sanitárias. Medidas de prevenção e controle de infecção devem ser implementadas pelos profissionais que atuam nos serviços de saúde, para evitar ou reduzir ao máximo a transmissão de microrganismos durante qualquer assistência à saúde realizada ${ }^{(4)}$, principalmente no que se refere ao quarto de idosos suspeitos ou confirmados da doença ${ }^{(5)}$.

Em epidemias ou pandemias, a área externa à residência deverá ser considerada contaminada e a área interna deverá ser considerada vulnerável. Deve-se escolher uma porta de entrada e estabelecer a área de transição em suas proximidades, no exterior ou no interior da residência ${ }^{(7)}$.

Dentre os fatores que provavelmente contribuem para a vulnerabilidade nestas instituições, inclui funcionários que haviam trabalhado enquanto eram sintomáticos; funcionários que trabalharam em mais de uma instalação; familiaridade e aderência inadequadas às recomendações de EPI; desafios para a implementação de práticas adequadas de controle de infecção(2), o que indica a necessidade de seleção de profissionais específicos para atuarem somente nesses quartos e a educação em saúde constante.

Idosos com quadro suspeito ou diagnóstico da COVID-19 devem ser isolados em quartos individuais, com porta fechada e bem ventilados, com janelas abertas ${ }^{(4,6)}$. Este é um local de grande investimento na prevenção de novos casos, necessitando de ações para impedir a disseminação do vírus em todo o ambiente.

Algumas ILPIs já dispõem de quarto para isolamento, entretanto, o número de casos pode aumentar e o número disponível não atender a demanda. Estudo aponta instituição que fez plano de contingência para pacientes com sintomas respiratórios e pneumonia em enfermarias designadas, caso os casos exceda a capacidade das instalações de isolamento(2).

Caso a ILPI não disponha de quarto para isolamento, se deve manter a distância de dois metros entre as camas ${ }^{(4)}$, colocando o menor número possível de idosos em cada quarto. Na entrada do quarto deve ser colocado o aviso de isolamento, bem como os EPI necessários no ambiente, devendo a ILPI garanti-los para todos os profissionais que tiverem contato nesse quarto ${ }^{(4)}$.

Os profissionais e cuidadores que entrarem no quarto dos residentes com infecção suspeita ou diagnóstico confirmado da COVID-19 devem ser orientados quanto à necessidade do uso de EPI, bem como devem ser capacitados sobre as técnicas de higiene das mãos, colocação e retirada dos EPIs ${ }^{(6)}$. Ao realizar higiene das mãos com água e sabão, utilizar, preferencialmente, toalhas de papel descartáveis para secar as mãos. Caso toalhas de papel descartáveis não estejam disponíveis, usar toalhas de pano e trocar quando ficarem molhadas ${ }^{(7)}$.

A proteção dos profissionais de saúde é uma prioridade(3). Para o cuidado ao idoso em quarto de isolamento, os EPIs necessários são: máscara cirúrgica, óculos de proteção, avental ou capote e luva de procedimento, porém, nem todas as ILPIs dispõem de todos esses equipamentos.

Após a saída do quarto, enfermaria ou área de isolamento, os EPIs devem ser imediatamente removidos ${ }^{(7)}$. Porém, caso o profissional de saúde saia de um quarto, enfermaria ou área de isolamento para atendimento de outro paciente com suspeita ou confirmação de infecção pelo novo coronavírus, na mesma área/setor 
de isolamento, logo em seguida, não há necessidade de trocar gorro (quando necessário utilizar), óculos/ protetor facial e máscara, somente avental e luvas, além de realizar a higiene de mãos ${ }^{(4)}$.

A orientação dos profissionais sobre o uso dos EPIs deve ser rotina nas ILPIs, tendo em vista a importância do uso adequado na prevenção e controle do coronavírus. Entretanto, estudo refere familiaridade e aderência inadequada às recomendações de EPI por profissionais que atuam em ILPIs ${ }^{(2)}$.

Ao entrar no quarto, que deve estar sempre com a porta fechada, deve ser criada uma área de transição. Esta área precisa estar bem definida, podendo-se delimitar o local com fita adesiva de cor contrastante no piso, ou mobiliário que permita a definição do espaço protegido(8).

Todos os materiais dentro do quarto isolado são considerados contaminados ${ }^{(8)}$. As superfícies das maçanetas de portas, mesas de cabeceira, cadeiras, armários, televisão e controles devem ser limpas várias vezes ao dia, com um produto de limpeza desinfetante, particularmente as superfícies mais utilizadas ${ }^{(5)}$. Os equipamentos reutilizáveis, a exemplo de termômetros, esfigmomanômetro e estetoscópio deverão ser adequadamente limpos e desinfetados antes e após o uso(5).

Os idosos devem ser orientados a não compartilhar objetos de uso pessoal como cortadores de unhas, alicates, esmaltes, escovas, pentes, aparelhos de barbear, celulares, tablet, mouse, sabonetes, toalhas, pratos, copos e talheres ${ }^{(6)}$. Entretanto, a realidade das ILPIs é a presença de elevado número de pacientes com demências, que podem não seguir essas orientações e usar objetos de outros idosos, caso compartilhem o mesmo quarto.

Ao utilizar o banheiro, deve ser feita a limpeza geral do local. As roupas devem ser retiradas com o mínimo de agitação e manuseio no quarto, devendo ser colocadas dentro de saco plástico e encaminhadas para máquina de lavar, e lavadas separadamente das roupas dos demais residentes. Os profissionais devem usar EPI para esse procedimento ${ }^{(5)}$.

As ILPI precisam reforçar a adesão da equipe às práticas de prevenção e controle de infecções, com observação do fluxo de trabalho da equipe. Interrupções substanciais, como absenteísmo da equipe e aumento da carga de trabalho, podem afetar a consistência com a qual essas práticas são implementadas e monitoradas ${ }^{(2)}$.

\section{Limitações}

Como o tema é atual, ainda dispõe-se de poucos estudos para ampliar a discussão, especialmente no contexto das ILPIs.

\section{Contribuições do ensaio reflexivo para a Enfermagem}

Destaca-se como contribuições desse estudo para a área da enfermagem a visibilidade de aspectos importantes na prevenção e controle da COVID-19 em ILPIs, que poderão nortear o planejamento da assistência e monitoramento dos residentes e profissionais.

\section{CONSIDERAÇÕES FINAIS}

No atual panorama de pandemia da COVID-19, o cuidado de enfermagem planejado para idosos que vivem em ILPIs abrange desde a porta de entrada a instituição, durante a sua permanência, até a saída dos profissionais envolvidos no cuidado, para evitar a propagação do vírus e manifestação da doença nos residentes.

\section{AGRADECIMENTO}

Ao Departamento Científico de Enfermagem Gerontológica da ABEn Nacional. 


\section{REFERÊNCIAS}

1. Organização Pan-Americana da Saúde (OPAS). Folha informativa - COVID-19 (doença causada pelo novo coronavírus). [Internet]. 2020. Disponível em: https://www.paho.org/bra/index.php?option=com_content\&view=article\&id=6101:covid 19\&Itemid $=875$

2. McMichael TM, Currie DW, Clark S. et al. Epidemiology of Covid-19 in a Long-Term Care Facility in King County, Washington. The new england journal of medicine [Internet]. 2020 [cited 2020 Apr 12]. Available from: https://www.nejm.org/doi/ full/10.1056/NEJMoa2005412

3. Tan LF, Seetharaman S. Preventing the Spread of COVID-19 to nursing homes. Experience from a Singapore Geriatric Center. J Am Geriatr Soc. [Internet]. 2020 [2020 Apr 16]. Available from: https://www.ncbi.nlm.nih.gov/pubmed/32216132

4. Agência nacional de vigilância sanitária (ANVISA). Nota Técnica GVIMS/GGTES/ANVISA № 04/2020. Orientações para serviços de saúde: medidas de prevenção e controle que devem ser adotadas durante a assistência aos casos suspeitos ou confirmados de infecção pelo novo coronavírus (SARS-CoV-2). (atualizada em 31/03/2020) [Internet] 2020. [Acesso 2020 Apr 08]. Disponível em: http://portal.anvisa.gov.br/documents/33852/271858/ Nota+T\%C3\%A9cnica+n+04-2020+GVIMS-GGTES-ANVISA/ab598660-3de4-4f14-8e6f-b9341c196b28.

5. Agência nacional de vigilância sanitária (ANVISA). Nota técnica GVIMS/GGTES/ANVISA № 05/2020. Orientações para a prevenção e o controle de infecções pelo novo coronavírus (sars-cov-2) em instituições de longa permanência para idosos (ILPI). [Internet]. 2020 [cited 2020 Abr 12]. Disponível em: http://portal.anvisa.gov.br/documents/219201/4340788/ NOTA+TE\%CC\%81CNICA+8+-+CORONAVI\%CC\%81RUS+2.pdf/75797abb-1bf5-4eb6-99e1-a1238269e30a

6. Brasil. Ministério da Saúde. Secretaria de Atenção Primária à Saúde. Nota Técnica № 8/2020-COSAPI/CGCIVI/DAPES/ SAPS/MS. 2020. [Acesso em 2020 Abr 08]. Disponível em http://189.28.128.100/dab/docs/portaldab/documentos/ notatecnica82020COSAPICGCIVIDAPESSAPSMS02abr2020COVID-19.pdf.

7. Brasil, MS. Nota Técnica № 9/2020-CGAHD/DAHU/SAES/MS. Recomendações da Coordenação-Geral de Atenção Hospitalar e Domiciliar em relação à atuação dos Serviços de Atenção Domiciliar (SAD) - Programa Melhor em casa na pandemia do Coronavírus (COVID-19). [Internet] 2020 [Acesso 2020 Abr 19]. Disponível em: https://www.saude.gov.br/images/ pdf/2020/marco/24/NOTA-T--CNICA-N----9-2020-CGAHD-DAHU-SAES.pdf

8. Brasil, MS. GVIMS/GGTES/ANVISA. Orientações da arquitetura hospitalar para controle de contágio: Covid19. [Internet] 2020 [Acesso 2020 Abr 18]. Disponível em: https://www.caubr.gov.br/wp-content/uploads/2020/04/COVID-19_ orientac\%CC\%A7o\%CC\%83es-ambiente-residencial-1.pdf 